\title{
El juego en red o el juego enredado: la dimensión interactiva de los juegos de las redes sociales
}

\author{
Carolina Duek \& Gastón Tourn \\ CONICET, Universidad de Buenos Aires / Oxford University, Google \\ E-mail: duekcarolina@gmail.com/gaston.tourn@gmail.com
}

\begin{abstract}
RESUMEN
El objetivo de este trabajo es presentar un análisis de los videojuegos de las redes sociales, en un contexto en el que gran parte de las interacciones digitales se despliegan en estas plataformas. Nos preguntaremos qué características particulares adquieren estos juegos para acercarnos a su sentido en el entramado cultural contemporáneo. De este modo, nos proponemos comprender qué tipos de in-

teracciones sociales se promueven y qué implicancias tiene esta modalidad de interacción en las prácticas lúdicas y sociales en general. Este análisis nos permitirá reflexionar sobre una arista poco explorada de las redes sociales, lo cual nos abrirá nuevos interrogantes para pensar las interacciones digitales y las tecnologías de la información y la comunicación (TICs).
\end{abstract}

Descriptores: redes sociales; videojuegos; TICs; interacciones digitales; comunicación.

Data de submissão: 04-05-2016. Data de aprovação: 23-10-2016.

A Revista Estudos em Comunicação é financiada por Fundos FEDER através do Programa Operacional Factores de Competitividade - COMPETE e por Fundos Nacionais através da FCT - Fundação para a Ciência e a Tecnologia no âmbito do projeto Comunicação, Filosofia e Humanidades (LabCom.IFP) UID/CCI/00661/2013. 


\title{
Network games or entangled games? The interactive di- mension of social games
}

\begin{abstract}
The objective of this paper is to present an analysis of social games in a context in which social media occupies a great part of digital interactions. We inquire which specific characteristics have these games to approach their meaning in the contemporary cultural framework. In this sense, we research which types of social

and what implications this type of interaction has in ludic and social practices in general. This analysis will allow us to reflect on an unexplored edge of social networks, which will open new questions to think about digital interactions and information \& communication technologies (ICT).
\end{abstract} interactions are promoted in these games

Keywords: social media; video games; ICT; digital interactions; communication.

\section{Presentación}

Las redes sociales han adquirido una importancia cada vez mayor en las discusiones sobre nuevas Tecnologías de la Información y la Comunicación (TICs). Resulta indudable que estas páginas de Internet empezaron a acompañar - desde hace unos años - las palpitaciones de nuestra vida cotidiana. Las estadísticas lo corroboran. Facebook, la red social más popular en la actualidad, es el tercer sitio web más visitado luego de Google y YouTube. Una visita típica a la plataforma de Zuckerberg dura alrededor de 20 minutos. Según la consultora comScore, Argentina lidera a nivel mundial el uso de redes sociales, con un consumo de casi 10 horas mensuales por usuario. América Latina destina a este tipo de páginas un 56\% más de tiempo que el promedio global.

En este contexto en el que proliferan las redes sociales, una arista poco explorada - en la bibliografía específica - ha sido el rol que desempeñan los videojuegos en el desarrollo de estas plataformas digitales. La reciente emergencia de diversas investigaciones en este novedoso campo de estudio se vio acompañada por el crecimiento exponencial de este fenómeno en línea. Actualmente, jugar es la quinta actividad más popular de Facebook. Asimismo, 
de las veinte aplicaciones más utilizadas y mejor calificadas en el sitio, dieciocho son videojuegos. Si bien estos juegos electrónicos no se propagan con la rapidez que alcanzan otras herramientas de esta red social, logran una lealtad extraordinaria ya que una vez que un usuario empieza a jugar, tiende a volver asiduamente.

A su vez, algunas de las redes sociales con mayor uso por fuera de Facebook se focalizan casi exclusivamente en el desarrollo de social games [juegos sociales], es decir, videojuegos inscriptos en redes sociales. En el caso argentino, Mundo Gaturro y Club Penguin constituyen dos sitios de referencia a la hora de jugar en Internet. Mundo Gaturro es una plataforma basada en los personajes del humorista gráfico Cristian Dzwonik (Nik) y financiada por la Compañía de Medios Digitales (CMD, empresa del Grupo Clarín S.A.). Club Penguin -creado en octubre de 2005- es una web que se asienta en un mundo virtual desarrollado por New Horizon Interactive (empresa que fue adquirida por The Walt Disney Company en agosto de 2007). Los personajes principales son unos pingüinos que participan en distintos juegos en un entorno similar a la Antártida. Ambas páginas priorizan en sus plataformas los juegos sobre cualquier otra práctica digital.

Si se tiene en cuenta que los juegos constituyen una de las actividades principales en Internet, resulta imprescindible analizar qué características particulares adquieren los mismos en tanto interacción virtual.

El propósito de este trabajo consiste en identificar qué tipo de relaciones, acciones y dinámicas se promueven en los videojuegos de las redes sociales mencionadas e identificar el rol que tienen, para los informantes, estos nuevos espacios de juego. De este modo, buscaremos reflexionar sobre las implicancias de estas interacciones digitales en las prácticas lúdicas y sociales. Asimismo, creemos que este análisis nos abrirá nuevos interrogantes para pensar las interacciones digitales y las TICs.

\section{ESTADO DE LA CUESTIÓN E HIPÓTESIS}

La velocidad con la que se han desarrollado los nuevos dispositivos electrónicos y medios de comunicación ha cambiado el ecosistema comunicativo (Martín-Barbero, 2003) en el que vivimos. Una de las transformaciones más importantes en relación a los vínculos son las redes sociales. Facebook, Twitter, LinkedIn, Instagram, Snapchat, entre muchas otras, han transformado el 
vínculo entre los sujetos, la tecnología y las formas de interacción. Hay varias razones por las que sostenemos esta afirmación. En primer lugar, la transformación de la escala y de la percepción del tiempo y el espacio (Jameson, 1998: VI-XVI) ha modificado las posibilidades de estar en contacto con diferentes personas en todo el mundo. Esto significa que, con un par de clics, podemos conectarnos con alguien del otro lado del planeta. Podemos encontrarnos antiguos compañeros, amigos, compañeros de escuela o ex novios. El contacto social es, en síntesis, la primera transformación.

En segundo lugar, las redes sociales se han convertido en la forma más eficaz de presentarse ante los demás (en el sentido propuesto por Goffman, 1974). A través de la interacción de todos los perfiles, imágenes y pensamientos publicados, configuramos una cierta imagen de nosotros mismos delante de nuestros "amigos"(Livingstone, 2009; Elias, 2015: 133-8).

En tercer lugar, el uso de la palabra "amigo"como sinónimo de añadir a alguien a nuestro perfil haciendo clic un par de veces (Turkle, 2015: 137-176). La idea de amigo, como sostiene Pahl (2003), necesita ser contextualizada ya que la amistad es un concepto informal, dinámico y variable. El tipo de amigos y las relaciones que (podemos) construir con ellos están íntimamente vinculados a las condiciones materiales y simbólicas en las que vivimos. Los amigos explican nuestras vidas y sus cambios. Estamos frente a un nuevo uso de la palabra: un amigo puede ser alguien que nunca conocimos, pero que es un conocido de un conocido en las redes sociales (Telles, 2011).

Fundamentaremos nuestro análisis en el interaccionismo simbólico y la teoría de la comunicación humana. Según Watzlawick et al. (1973), la comunicación posee ciertas propiedades de naturaleza axiomática que llevan implícitas consecuencias fundamentales para el estudio de las interacciones. Watzlawick et al. enumeran cinco axiomas de la comunicación.

1. No es posible no comunicar.

2. Toda comunicación tiene un nivel de contenido y un nivel relacional.

3. La naturaleza de una relación depende de la forma de puntuar las secuencias de comunicación (y del vínculo entre los interlocutores).

4. Las personas utilizan tanto la comunicación digital como la analógica (verbal y no verbal).

5. Todos los intercambios comunicacionales son simétricos o complementarios, según estén basados en la igualdad o en la diferencia. 
Basándonos en algunas dimensiones de estos postulados, analizaremos qué tipos de interacciones se promueven en los juegos de las redes sociales y qué implicancias tienen las nuevas modalidades de interacción en las prácticas lúdicas y sociales en general.

La hipótesis que vamos a sostener en este trabajo es que los social games se construyen como espacios que permiten - a diferencia de los videojuegos tradicionales - conocer nuevas personas y mantener el contacto con "amigos". Si bien estos videojuegos se definen como "sociales", planteamos que la interacción se encuentra restringida por la arquitectura de estas plataformas digitales. A su vez, nos preguntaremos si los intercambios que se promueven en estos videojuegos se asientan sobre mecanismos lúdicos o de contacto "virtual" y si son vínculos recíprocos o competitivos.

En primer lugar, sugerimos que los social games establecen la acumulación de objetos y el exhibicionismo como principales dinámicas de interacción. En este sentido, la mecánica de estas plataformas pareciera operar principalmente como un medio para la institución y la preservación del status, a través de la ostentación de diversos consumos mediante la conformación "automática" de rankings.

En segundo lugar, creemos que la mayor parte de estas características no son exclusivas de las aplicaciones de las redes sociales, sino que replican ciertos esquemas culturales imperantes. Los social games no reemplazarían a los juegos y videojuegos previos, sino que entrañan la supervivencia de rasgos de ciertos juegos "tradicionales". Las prácticas lúdicas en las plataformas virtuales parecieran reciclar algunos elementos del entramado cultural contemporáneo y exacerbar ciertos patrones ligados al consumo, la acumulación y la exhibición.

Para Vigotsky ([1930] 1986: 12), los juegos son una "escuela vivencial" para los niños y niñas. Su importancia es vital para modelar la personalidad de los más chicos y la forma en la que se vinculan con el mundo. En la actualidad, el lugar de los juegos se multiplica por la disponibilidad y portabilidad de los dispositivos, pero, también, por la existencia de plataformas y redes sociales que alientan las interacciones lúdicas a través de diferentes propuestas. Es por ello que analizar los juegos y las interacciones a través de las nuevas tecnologías no sólo nos abre un campo de estudio sobre el juego, sino que da cuenta de la relevancia de los temas y problemas de la cultura infantil contemporánea (Steinberg y Kincheloe, 1997: 26). 
Por último, sugerimos que la creciente interacción por medios digitales reconfigura los modos de sociabilidad contemporáneos, ya que estas prácticas virtuales forman parte de una transformación que tiende a la vinculación por medio de pantallas (Jenkins et al., 2015).

\section{MATERIAL Y MÉTODOS}

La investigación en la que se enmarca este trabajo - y el estudio específico que desarrollaremos aquí de un fenómeno cultural contemporáneo - se propone articular diferentes fuentes y métodos de investigación. Mediante el andamiaje metodológico que se precisa a continuación, buscamos construir distintas interpretaciones que den cuenta de la complejidad que presentan los social games.

\section{Análisis de juegos}

Relevamos la oferta disponible de social games en Facebook, Mundo Gaturro y Club Penguin e identificamos y analizamos los principales videojuegos. Los social games más significativos fueron caracterizados a partir de una grilla que consideraba los siguientes criterios: a) objetivo; b) diseñador; c) empresa desarrolladora; d) año de lanzamiento; e) plataforma; f) género; g) multijugador cooperativo/competitivo; h) rol protagónico; i) rol del usuario; j) presencia o ausencia de niveles; k) tipos de escenarios y l) propagación a otros soportes como bandas de sonido, films, merchandising, etc. (para ampliar, ver Tourn, 2013).

En este trabajo no nos centraremos en los videojuegos de consola dado que trascienden a los objetivos del presente análisis. Un estudio exhaustivo de esos videojuegos puede consultarse en Duek (2012) y Tourn (2012).

A partir de la comparación de las dinámicas, los roles, los personajes, las temáticas y las acciones de los principales videojuegos de las redes sociales, construimos una tipología general de estas prácticas lúdicas.

\section{Entrevistas en profundidad}

Para indagar sobre las prácticas de los sujetos en estas plataformas virtuales, realizamos una serie de entrevistas en profundidad en el proyecto de 
investigación PICT 2011-2013: "Juguetes, consolas y dispositivos electrónicos: ¿los nuevos auxiliares lúdicos infantiles? (un análisis del juego infantil contemporáneo)", dirigido por la Dra. Carolina Duek (UBA/CONICET). Entrevistamos a veintidós informantes de entre 6 y 11 años, que cumplieran con los siguientes requisitos: 1) ser habitantes de la Ciudad de Buenos Aires, 2) estudiar en escuelas públicas de jornada simple, y 3 ) utilizar redes sociales. Teniendo en cuenta que la población a analizar era demasiado amplia, construimos una muestra bajo los criterios de saturación teórica (Glaser y Strauss, 1967: 61).

En estas entrevistas, distinguimos los usos de las redes sociales que hacen los informantes. En esta línea, rastreamos los contextos en los que las usan, si lo hacen solos o acompañados, fuera o dentro del horario escolar, etc. Asimismo, examinamos los tipos de plataformas que prefieren y qué placeres y displaceres encuentran en esta práctica. Mediante este relevamiento, identificamos el lugar que ocupan las redes sociales en la población estudiada.

En este trabajo, no abordaremos las interacciones que suceden en los foros de los social games debido a limitaciones de espacio. Un análisis pormenorizado de estas comunidades digitales puede consultarse en Tourn (2012).

\section{Análisis de discurso}

Luego de examinar los videojuegos de las redes sociales y entrevistar a sus usuarios, identificamos las semejanzas y diferencias entre los usos y apropiaciones de las distintas plataformas. De este modo, observamos las tácticas (en el sentido descripto por De Certeau, 1996: 68) que se llevan a cabo en estas prácticas digitales. Por otra parte, realizamos una descripción de distintos discursos que propician el consumo de niños y niñas, a partir del herramental semiótico de la Teoría de los Discursos Sociales (Verón, 1985). Por medio de este análisis, indagamos el lugar que ocupan las redes sociales en el marco general de las redes de consumo para el mercado infantil. Este ejercicio nos permitió reflexionar críticamente sobre las plataformas que promueven hábitos de consumo en niños de entre 6 y 10 años.

Mediante la utilización de estos métodos y técnicas de investigación, buscamos revisar las hipótesis iniciales y rediscutir ciertos aspectos teóricos del campo de la comunicación y la cultura. 
En el próximo apartado, analizaremos en detalle las interacciones que los niños tienen con los videojuegos de las redes sociales. Identificaremos qué tipos de relaciones, acciones y dinámicas se promueven en los social games.

\section{ANÁlisis Y RESULTADOS}

El abordaje de los resultados de la investigación nos demandó la operacionalización de los cinco axiomas de Watzlawick et al. (1973). En este artículo trabajaremos con algunos elementos de los axiomas que nos permitirán iluminar los resultados de la indagación sobre la que se organiza este trabajo. Probablemente sea el primero de todos el que organizará la presentación de los resultados: "No es posible no comunicar". Esto significa que todo acto es un acto de comunicación. Intencional, involuntario, arbitrario o convencional, somos seres comunicantes que, a su vez, no pueden optar por la no-comunicación. Analizar la relación entre los usuarios y las pantallas exige, en consecuencia, la pregunta por las formas que asumen tanto la interacción como la comunicación en un contexto signado por los accesos, los contactos y los "amigos" virtuales. La afirmación de la imposibilidad de la comunicación jerarquiza nuestro análisis al identificar todo acto (un clic, un "me gusta", una invitación a compartir un espacio) como una forma de comunicación en pos de un objetivo de interacción.

En el año 2006, el Ministerio de Educación de la Argentina condujo una encuesta nacional de consumos culturales y los resultados presentados sostienen que ningún niño o niña mencionó el uso de una red social como una práctica cotidiana (para ampliar los detalles de los resultados ver Morduchowicz, 2012). Seis años después, en 2012, una nueva investigación del Ministerio mostró que más del $95 \%$ de los encuestados visitaban esos sitios y que el $75 \%$ de ellos tenía un perfil activo en la red social Facebook, la página más visitada en la Argentina (Morduchowicz, 2012).

Una visita típica a la plataforma desarrollada por Mark Zuckerberg dura, aproximadamente 20 minutos ${ }^{1}$. En este contexto de proliferación y crecimiento exponencial del uso y de las apropiaciones de las redes sociales, el juego aparece como una dimensión poco explorada por la bibliografía, pero muy

1. Este dato fue relevado por Alexa, una compañía especializada en el análisis de tráfico web. Las métricas actualizadas sobre Facebook pueden examinarse en www.alexa.com/ siteinfo/facebook.com 
relevante en la palabra de los informantes que participaron en nuestra investigación. Jugar en Facebook es, como ya mencionamos, la quinta actividad más popular en la plataforma.

La consultora eMarketer (2013) sostiene que casi la mitad de los usuarios de Facebook utiliza la red para jugar, actividad que está por encima de "ver videos", otra de las actividades más frecuentes por parte de los usuarios. Finalmente, según cifras oficiales de Facebook, 18 de las 20 aplicaciones mejor calificadas son juegos ${ }^{2}$. Según Kirkpatrick (2010: 156), en los casos de juegos no masivos (con una acotada cantidad de jugadores), la fidelidad de los usuarios es tan extraordinaria que la relación con ese juego se sostiene en el tiempo. En síntesis, no se trata solo de analizar las estadísticas de los juegos en línea sino de ver de qué modos se sostiene la interacción con la plataforma en el sentido que Hall (1963) define la relación entre comunicación y espacio. Definir territorios, marcar límites utilizando todos los canales de comunicación disponibles, es un elemento común en la interacción contemporánea (Baylon y Mignot, 1996, Verón, 1987, Graner Ray, 2004). El territorio ha sido clave para el análisis de la comunicación pero, en la actualidad, se extiende, por medio de aparatos y dispositivos, al mundo virtual y se abre a nuevas formas de vinculación, conexión e intercambio.

Una de las invariantes que apareció en la palabra de los informantes se vincula con la socialización y con la puntuación, tal como la definen los axiomas de la comunicación, de las relaciones:

A mí me encantan los juegos en Facebook, me gustan todos (...) pero juego más a los que juegan mis amigos por los rankings y para que estemos todos haciendo algo parecido. No está bueno ser el único que juega a ese juego porque no puedo intercambiar cosas con nadie y no te ayudan nada.

(Tomás, 8 años)

Tomás sintetiza varias dimensiones que aparecieron con recurrencia en los testimonios de los informantes. Jugar es una actividad que identifica como placentera, pero hay una clave que va más allá de las peculiaridades del juego: la relación con otros a través del juego. Facebook propone, desde sus comienzos, una manera de organizar las relaciones virtuales bastante particular; no

2. Las cifras oficiales de Facebook pueden consultarse en www.facebook.com/appcenter 
se trata solamente de tener "amigos", sino que cada vez que habilitamos una aplicación o juego nuevo, nos muestra quiénes están jugándolo, cuáles son sus mayores puntajes y cómo se compone la tabla de resultados entre nuestros contactos. Es decir, jugar a un juego en la red social no se limita a esa actividad, sino que implica exponerse, exhibirse y competir. En ese sentido, Goffman (1974) identifica el trabajo de la cara [el facework] como una de las dimensiones a través de la cual los sujetos sociales construyen sus interacciones. Evalúan qué aspectos serán valorados por pares, qué beneficios pueden obtener y de qué formas pueden construirse vínculos en un espacio novedoso.

"No está bueno ser el único que juega", dice Tomás y despliega una gran cantidad de elementos a tener en cuenta a la hora de analizar la relación con la red social. Por un lado, la valoración del contacto con otros; por otro, la necesidad de colaborar con otros y de contar con su ayuda. Y es aquí donde aparece una de las claves ya no sólo de Facebook sino también de Mundo Gaturro y Club Penguin. Los tres sitios encabezan la lista de los más visitados por parte de niños y niñas de la Argentina (Duek, 2014), y se caracterizan por presentar juegos e interacciones que "necesitan" de un otro-usuario que contribuya al cumplimiento de los objetivos: en los tres espacios los juegos, los objetos y objetivos pueden lograrse por medio de "donaciones" de otros usuarios y se reciben atributos y elementos que sólo pueden enviarse por medio de vínculos virtuales. Por ejemplo, en Mundo Gaturro, la vestimenta más "exclusiva" no se compra sino que se "comparte" con otros jugadores. Al enviar a un "amigo" un objeto, inmediatamente el receptor debe responder de manera equivalente con un envío similar. Interacción, conveniencia e igualación son algunas de las claves para comprender las relaciones en estas plataformas.

Los juegos en redes sociales comparten características que los diferencian de otros tipos de juegos electrónicos. O’Neill (2008: 4) los define como:

Una actividad estructurada con reglas contextuales a través de las cuales los usuarios se vinculan. Los social games deben ser multi-jugadores y tener al menos una de las siguientes características: basados en turnos, en plataformas que brindan la posibilidad de componer un usuario temporal que interactúa con otros por el mismo canal de contacto.

Los social games desarrollados por Facebook, Mundo Gaturro y Club Penguin presentan patrones comunes en diferentes aspectos. En todas las pla- 
taformas, los jugadores encuentran mundos virtuales de simulación con reglas propias. Los sitios mencionados crean un entorno digital en el que los usuarios se vinculan unos con otros. En estos ambientes simulados, los usuarios pueden jugar diferentes videojuegos diseñados con gráficos simples y un grado de dificultad baja en términos de resolución de obstáculos. Los social games priorizan la simplicidad y la repetición de las acciones por sobre la complejidad y la innovación (Tourn, 2013: 37).

Sarlo (1994) define a los videojuegos como ficciones "de acción sin una historia" que se despliegan mientras se introduce un argumento en el que todo ocurre en términos de movimientos pero no queda nada en lo profundo del relato. Hay hilos conductores pero vinculados a la inmediatez de la resolución de conflictos y en pos de un fin, de una misión breve y no compleja (claro que esto no ocurre para todos los tipos de juego sino que se identifica claramente en los social games). Urresti (2008) agrega que este tipo de juegos requieren planeamiento y velocidad para resolver objetivos relativos al plano visual antes que a la dimensión lingüística: las acciones se "leen" desde las imágenes que se despliegan en la pantalla. Los social games en general no tienen diferentes niveles de complejidad y los usuarios los identifican de este modo:

Los juegos son una pavada. Pero me divierten. Me da risa cuando Gaturro se mueve como loco en la pantalla.

(Sofía, 9 años)

Si me preguntás por los juegos, son una porquería. Prefiero un millón de millones de veces los de la Play [Station] pero los de Gaturro son buenos porque están en Mundo Gaturro haciendo cosas todo el tiempo. Pero jueguitos posta, son los de la Play.

(Ulises, 9 años)

Los usuarios identifican claramente las diferencias entre los social games y los de las consolas. La PlayStation ${ }^{3}$ se vincula con la calidad de los juegos mientras que los social games se organizan en torno de la interacción con pares. Watzlawick et al. (1973) sostienen en uno de los axiomas que "toda

3. La PlayStation es una consola de juegos de Sony, la más popular en su segmento comercial con 15 millones de dispositivos vendidos en 2014, según la consultora económica VG CHARTZ. 
comunicación tiene un nivel de contenido y un nivel relacional" y es interesante reflexionar sobre la forma en la que los entrevistados han diferenciado, no sólo las plataformas, sino los espacios destinados a la interacción en y a través de ellas. Si los social games son espacios de socialización bajo la excusa del juego, los juegos en las consolas tienen otras particularidades, tramas y dinámicas que se relacionan más con el juego y sus características que con las formas de interacción a través de él.

En síntesis, los juegos en las redes sociales presentan una baja calidad en los gráficos y en sus términos narrativos comparados con otros productos de la industria de los videojuegos (cfr. Kafai et al., 2008 y Casell y Jenkins, 1998 para más precisiones). Es por ello que la experiencia de "jugabilidad" se vincula más a los llamados casual games que a los juegos de inmersión o de múltiples niveles. Los casual games son juegos en los que los objetivos son muy sencillos y no se articulan con otros niveles de dificultad. Pensemos, por ejemplo, en la "Carrera de embolsados" de Club Penguin o en "El juego de los penales" en Mundo Gaturro: en ambos, el objetivo es cumplir un objetivo simple [llegar a la meta o marcar un gol] y, una vez logrado, el juego termina y no tiene continuación.

Los casual games son juegos que demandan una baja dedicación por parte del jugador y una alta participación de la interacción entre usuarios dado que, mientras juegan, se habilitan chats entre quienes compiten, se permite conocer a los rivales, interactuar a través de movimientos en la pantalla o por medio del uso de frases o emoticones ${ }^{4}$. $\mathrm{Y}$ es aquí donde el juego como práctica queda desplazado por parte de las interacciones entre usuarios. No es tan importante lo que ocurre en el juego como aquello que pasa entre los jugadores.

Complementariamente, si Facebook se organiza en torno de los rankings de desempeño y sugiere acciones para intercambiar o ayudar a jugadores (como mencionaba Tomás, nuestro informante), en Mundo Gaturro y en Club Penguin la clave radica en la acumulación de puntos que se obtienen jugando. Los puntos sirven para comprar objetos en el mercado virtual de la plataforma. Ropa para los pingüinos (en Club Penguin) o para "Gaturro" o "Agatha" (en Mundo Gaturro) y accesorios o decoración para las "casas" de los personajes. La obtención de puntos es clave para la diferenciación: cuantos más puntos

4. Los emoticones son dibujos preexistentes que apuntan a transmitir emociones o pensamientos. 
obtiene un usuario, más "especial" es su personaje. Y, a la hora de interactuar con pares, se construye la diferencia de la que hablan Watzlawick et al. (1973) sobre las relaciones complementarias o simétricas. En las redes sociales y plataformas de juego que estamos analizando, el objetivo es imponerse ante los otros por medio de la seguridad que brindan, a los usuarios, las posesiones digitales de sus personajes. El personaje mejor vestido, el que tiene una tarjeta de crédito (virtual) VISA, un celular o algún auspicio en su casa, es aquel que tiende a ser más valorado por sus pares:

Facu tiene pasaporte y, ¿te digo? Es in-cre-í-ble la ropa de su Gaturro, la casa cómo la tiene (...) Todos quieren jugar con él porque ir a una fiesta en su casa es lo más de lo más (...) Y Facu cada vez invita a menos gente porque se cree el líder de todos.

(Marina, 8 años)

Una segunda dimensión de diferenciación aparece con los accesos privilegiados: tener una tarjeta de crédito vinculada a una cuenta de Facebook (y usarla para comprar vidas, crédito o lo que haga falta), pasaporte en Mundo Gaturro o ser socio en Club Penguin es una marca de "clase" al interior de las plataformas. Quienes abonan para ingresar (es decir, aquellos que no utilizan el modo "básico" de las plataformas) acceden a productos preferenciales, decoración peculiar y personajes deseados. Tal como plantean Revuelta y Esnaola (2012), las compañías desarrolladoras de social games hacen uso del modelo freemium ${ }^{5}$ para atraer a más usuarios.

Si bien todo ocurre en el mundo virtual, la exhibición aparece como clave a la hora de asumir roles de grupo (Turkle, 2011). Mostrar la ropa y los puntos obtenidos, saber quién es usuario "básico" y quién es "premium" es una manera de ingresar a un universo en el que la diferenciación y la asunción de roles específicos es la clave para comprender las interacciones entre pares. Cantora y Molinari (2012: 56) identificaron la competencia y la exhibición como las dos dimensiones más importantes en las plataformas de juego para niños y niñas:

5. Freemium es un modelo de negocios que funciona ofreciendo servicios básicos gratuitos, mientras se cobra por otros más avanzados o especiales. La palabra freemium es una contracción en inglés de las dos palabras que definen el modelo de negocios: "free" y "premium". 
Yo juego a Club Penguin porque todos mis amigos juegan ahí y no me sirve no conocer a nadie ni poder compartir ni mostrarle a los otros lo que hago, lo que hace mi personaje y lo que le compro.

(Sabrina, 9 años)

Sabrina (y los informantes de la muestra a los que ella representa) nos explica su relación con Club Penguin a partir de las siguientes dimensiones:

I. La relación con sus pares.

II. La importancia de que todos jueguen en la misma red o plataforma.

III. El rol de la exhibición como forma de interacción.

IV. Las compras que hace para su personaje.

V. La noción de utilidad ("no me sirve").

Las cinco claves del testimonio de la informante nos ubican en un universo en el que los juegos son una excusa para la socialización y la vinculación con pares. Jugar en Facebook, en "Club Penguin" o en "Mundo Gaturro aparece, en nuestros resultados de investigación, como un puente para reforzar lazos, roles, jerarquías y relaciones.

Una dimensión fundamental en este análisis es la presencia (o ausencia) de controles parentales en las diferentes plataformas digitales. Tanto Mundo Gaturro como Club Penguin establecen fuertes regulaciones respecto a la seguridad de los jugadores en Internet. En ambas plataformas, los niños deben ingresar la dirección de correo electrónico de sus padres al momento de registrarse. La cuenta no se activa hasta que el adulto acepte la participación del chico. A su vez, los padres deben elegir entre dos modos de seguridad que buscan limitar las interacciones posibles en la red social. En Mundo Gaturro, estas modalidades de acceso se denominan "Moderado" y "Lista Blanca":

- Moderado (Seguridad Media): el texto escrito es validado contra un conjunto de palabras prohibidas antes de ser mostrado y luego revisado por el equipo de moderadores. (Nota: el filtro de palabras prohibidas se actualiza constantemente, no obstante pueden filtrarse mensajes que ofendan a ciertos jugadores.)

- Lista Blanca (Seguridad Alta): limita lo que se puede escribir o leer a un conjunto predeterminado de textos (mensajes, saludos, preguntas, 
comentarios, etc.) seleccionables por menú, restringiendo la interacción solo a usuarios que elijan el mismo modo de chat.

(www.mundogaturro.com)

En Club Penguin, aparecen las mismas opciones y se denominan "Chat de seguridad estándar" y "Chat de alta seguridad", respectivamente. En todos los casos, los adultos están habilitados a revisar las interacciones que mantienen sus hijos. Asimismo, son notificados si el niño dice un insulto o grosería. Pese al rigor de estos controles parentales, todos los chicos entrevistados violan las disposiciones, al colocar sus propias casillas de correo, en lugar de la dirección de sus padres.

¿Escribiste el mail de tus papás?

Yo siempre puse el mío.

$¿$ Recibiste la nota de seguridad?

Sí, la firmé yo.

(Tomás, 8 años)

Por el contrario, Facebook no posee un modo de vigilancia estricto ni estipula diferentes tipos de seguridad. Asimismo, no permite que los adultos puedan observar la actividad de sus hijos en la web.

La legislación en materia de privacidad nos impide otorgar acceso a nadie que no sea el titular de la cuenta. Animamos a los padres a supervisar el uso que hacen sus hijos de Internet y proteger sus equipos.

(www.facebook.com/help)

Esta amplia ausencia puede deberse a que Facebook dispone - a diferencia de Mundo Gaturro y Club Penguin - que es obligatorio tener 13 años o más para unirse al sitio. Sin embargo, los chicos encontraron una vez más las formas de vulnerar esta regla: nuestros entrevistados se hicieron pasar por más grandes al momento de registrarse o utilizan las cuentas de hermanos mayores y otros adultos.

Empezamos el análisis con la presentación del primer axioma del interaccionismo: "No es posible no comunicar". Hemos recorrido algunos aspectos de los otros axiomas y lo que encontramos es que muchos de los postulados 
que vinculan comunicación e interacción se conjugan nuevamente en las nuevas redes y plataformas. Los social games nos brindan aspectos de la relación entre pares, de los vínculos con los padres, de las formas diversas de la comunicación multicanalizada (digital/verbal, analógica/no verbal), la puntuación de la relación y la simetría o complementariedad de los intercambios.

La investigación se organizó en torno de la pregunta por los juegos y las nuevas tecnologías y lo que encontramos, en la indagación, son diferentes formas de caracterizar y de nombrar la interacción a través de las nuevas plataformas y redes sociales. García y Monferrer (2009: 84) sostienen que la comunicación no es el modo de recibir la cultura sino el instrumento utilizado para su construcción y es en esa línea en la que se ordenarán nuestras conclusiones.

\section{DiscuSión DE RESULTADOS}

Este trabajo comenzó con la presentación de un nuevo espacio lúdico para los niños y las niñas y se orientó hacia la identificación de las formas de interacción convocando la palabra de los entrevistados. Indagamos las relaciones, las dinámicas y las acciones que suponen los social games para ver, en ellos, las maneras en las que se caracterizan los juegos, las plataformas y las formas de conexión con pares. La hipótesis del artículo sostenía que los social games se construyen como espacios para conocer nuevas personas y mantener el contacto con "amigos" virtuales.

Hemos identificado, a lo largo de la investigación, la importancia que tiene para los niños y las niñas la pertenencia a espacios comunes con sus pares. Un informante ya citado mencionó la importancia de jugar a los juegos que sus amigos eligen porque "no me sirve no conocer a nadie". Esta afirmación sintetiza gran parte de los hallazgos sistematizados: los social games no se valoran por las características propias del juego sino por los pares que los eligen y convierten en una "tendencia" de ese grupo. Una vez elegido un juego - y dado que todos los "amigos" que juegan se ubican en rankings por nivel de performance - comienza un tipo de interacción que se vincula específicamente a las demandas del juego (el "envío" de objetos necesarios o deseados) y con la exhibición de los logros obtenidos. Mencionamos que los logros pueden relacionarse con los puntajes obtenidos y con los objetos y vestimentas que tenga nuestro personaje en las plataformas de juego. La mirada de los 
otros se ordena, también, por la valoración esperada de terceros de los logros alcanzados. Es, en un punto, la reactualización de la tradicional frase "mirá, [destinatario: madre, padre, amigos, docentes] lo que soy capaz de hacer", pero multiplicada a todos los contactos que ven las publicaciones y se vinculan por medio de las plataformas mencionadas (Duek, 2014).

Nos preguntamos al comienzo si los mecanismos de intercambio se organizaban en torno del valor lúdico de los social games o si prevalecía el eje del contacto y la definición de las relaciones con pares. Encontramos, en la investigación, que lo más relevante para los informantes se relacionaba con seguir las tendencias pero, también, con la importancia de mantener abiertos todos los canales de contacto.

Quedarse fuera de las interacciones aparece como una gran amenaza que debe ser minimizada. Es por ello que "jugar a lo que todos juegan" no tiene tanto que ver con la valoración de los juegos y sus características lúdicas, sino con los pares que se suman a dichas interacciones. Es conveniente, a los fines de la obtención de puntos, contar con personas que envíen objetos o regalos en la plataforma del juego pero lo es, también, saber que todo lo que ocurre (y es valorado por el jugador) puede ser exhibido no sólo en el sitio específico sino en todas las redes sociales vinculadas (hay un botón de "compartir" que extiende los desempeños y exhibiciones en una plataforma a Facebook y Twitter).

\section{CONCLUSIÓN}

Exhibir, competir, valorar y ser valorado son cuatro de las claves que identificamos en nuestro trabajo. La escuela de Palo Alto define, en su primer axioma, la imposibilidad de no comunicar. Si no es posible no comunicar, en época de redes sociales y de conexión, cada clic, cada intercambio, sugerencia o emoticón se develan cruciales para la puntuación de la relación, para la definición del vínculo (complementario o simétrico) pero, también, para la construcción de una imagen de nosotros mismos frente a los otros. ¿Quiénes somos?, ¿cómo elegimos mostrarnos?, ¿qué acciones imaginamos serán valoradas por terceros?, ¿qué perfil asumimos? Estas preguntas constituyen, para nosotros, las claves para el análisis de las interacciones contemporáneas. Y serán las respuestas (temporales y provisorias) que podamos construir por medio de la investigación científica, las que nos ayudarán a definir, en diferentes 
momentos de la historia, las dinámicas, acciones y relaciones que pueden (o no) construirse en los diferentes espacios de la vida social.

\section{REFERENCIAS BIBLIOGRÁFICAS}

Baylon, C. \& Mignot, X. (1996). La comunicación. Madrid: Cátedra.

Cantora, A. \& Molinari, L. (2012). Jugar a "Mundo Gaturro". Acerca del juego, la exhibición y el consumo en la plataforma online. Tesina de licenciatura en Ciencias de la Comunicación, Facultad de Ciencias Sociales, Universidad de Buenos Aires.

Cassell, J. \& Jenkins, H. (2000). Chess for girls? feminism and computer games. In From Barbie to Mortal Kombat. Gender and computer games, Boston: MIT Press.

De Certeau, M. (1996). La invención de lo cotidiano I. Artes de hacer. México: Universidad Iberoamericana.

Duek, C. (2012). El juego y los medios. Autitos, muñecas, televisión y consolas. Buenos Aires: Prometeo Libros.

Duek, C. (2014). Juegos, juguetes y nuevas tecnologías. Buenos Aires: Capital Intelectual.

Elias, H. (2015) Brandware. When brands and digital media collide. Consultado en www.herlanderelias.com

García, M. \& Monferrer, J. (2009). Propuesta de análisis teórico sobre el uso del teléfono móvil en adolescentes. Comunicar, 33. Huelva: Grupo Comunicar.

Glaser, B. \& Strauss, A. (1967). Discovery of grounded theory: strategies for qualitative research. Chicago: Aldine Publishing Company.

Goffman, E. (1974). Frame analysis. Boston: Northeastern University Press.

Graner Ray, S. (2004). Gender inclusive game design: expanding the market. Hingham: Charles River Media.

Hall, E. (1963). Proxemics: the study of man's spatial relations. In Galdston (ed.) Man's image in medicine and in anthropology. Nueva York: International Universities Presses. 
Jameson, F. (1998). Preface. In Jameson \& Mayoshi (eds.) The cultures of globalization. Duke University Press.

Jenkins, H.; Ito, M. \& Boyd, D. (2015). Participatory culture in a networked era: a conversation on youth, learning, commerce and politics. Cambridge: Polity Press.

Kafai, Y., Heeter, C., Denner, J. \& Sun, J. (2008). Beyond Barbie and Mortal Kombat: new perspectives on gender and gaming. Boston: MIT Press.

Kirkpatrick, D. (2010). The Facebook effect. New York: Simon \& Schuster.

Livingstone S. (2009). Children and the Internet. Cambridge: Polity.

Marc, E. \& Picard, D. (1992). La interacción social: cultura, instituciones y comunicación. Barcelona: Paidós.

Martín-Barbero, J. (2003). Retos culturales de la comunicación a la educación: elementos para una reflexión que está por comenzar. In R. Morduchowicz (comp.) Comunicación, medios y educación. Barcelona: Octaedro.

Morduchowicz, R. (2012). Los adolescentes y las redes sociales. Buenos Aires: FCE.

O'Neill, N. (2008). What exactly are social games?. Consultado en www.soci altimes.com/2008/07/social-games

Pahl, R. (2003). Sobre la amistad. Madrid: Siglo XXI.

Revuelta, F. \& Esnaola, G. (2012). Videojuegos en redes sociales: perspectivas del edutainment y la pedagogía lúdica en el aula. Barcelona: Laertes.

Sarlo, B. (1994). Escenas de la vida posmoderna. Buenos Aires: Ariel.

Steinberg, S. \& Kincheloe, J. (1997). Cultura infantil y multinacionales. Madrid: Morata.

Telles, A. (2010). A revoluçao das mídias sociais. San Pablo: M.books.

Tourn, G. (2012). Ludomorfosis: una reflexión sobre la dimensión lúdica de los videojuegos. Question, primavera, 36.

Tourn, G. (2013). Del face to face al face to Facebook: redes sociales, videojuegos e interacciones digitales. Tesina de licenciatura en Ciencias de la Comunicación, Facultad de Ciencias Sociales, Universidad de Buenos Aires. 
Turkle, S. (2011). Alone together: why we expect more from technology and less from each other. Boston: MIT Press.

Turkle, S. (2015). Reclaiming conversation: the power of talk in a digital age. New York: Penguin.

Urresti, M. (2008). Ciberculturas juveniles. Buenos Aires: La Crujía.

Verón, E. (1987). Cuerpo y metacuerpo en democracia audiovisual. París: Après.

Vigotsky, L. S. ([1930] 1986). La imaginación y el arte en la infancia. Madrid: Akal.

Wark, M. (2007). Gamer theory. Cambridge: Harvard University Press.

Watzlawick, P.; Helmick Beavin, J. \& Jackson, D. (1973). Algunos axiomas exploratorios de la comunicación humana. In Teoría de la comunicación humana. Buenos Aires: Tiempo Contemporáneo.

\section{Juegos electrónicos}

Farmville

Creador: Zynga

Plataforma: Facebook

Año de lanzamiento: 2009

Pet Society

Creador: Playfish, Electronic Arts

Plataforma: Facebook

Año de lanzamiento: 2009

Mundo Gaturro

Creador: Compañía de Medios Digitales, Grupo Clarín

Plataforma: Mundo Gaturro

Año de lanzamiento: 2010

Club Penguin

Creador: Disney Interactive Solutions

Plataforma: Club Penguin

Año de lanzamiento: 2005 